\title{
Stability Criteria for a Polydisperse System
}

\author{
Jun Cai and John M. Prausnitz* \\ Department of Chemical Engineering, University of California, Berkeley and \\ Chemical Sciences Division, Lawrence Berkeley National Laboratory, Berkeley, CA94720
}

\begin{abstract}
Stability theory for fluid mixtures is used to calculate the spinodal and the critical locus for a system containing one discrete light component and one continuously distributed heavy "component". For reduction to practice, the criteria developed here are suitable for molecularthermodynamic models where the model parameters depend on the distribution variable that characterizes the continuous "component". To illustrate, the Soave-Redlich-Kwong equation of state is used to calculate critical properties of alkane mixtures where methane $\left(\mathrm{C}_{1}\right)$ is the discrete component and $\mathrm{C}_{2}-\mathrm{C}_{16}$ comprise a continuous "component". Calculated results show that, while systems containing methane and primarily small paraffins show a Type I critical locus, those containing methane and appreciable amounts of large paraffins show a Type V critical locus. These results are consistent with experimental critical-locus data for $\mathrm{C}_{1}-\mathrm{C}_{3}$ (Type I) and those for $\mathrm{C}_{1}-\mathrm{C}_{6}$ (Type V).
\end{abstract}

\section{Introduction}

In typical industrial chemical processes, the number of components is very large; well-known examples are crude oil or natural gas. It is often not practical, indeed it may be impossible, to perform the detailed chemical analysis to determine the exact compositions of such systems. In some cases, chemical analysis can provide only a few statistical properties, such as molar-mass distribution, normal-boiling-point distribution and so on. Continuous thermodynamics [1-10] has been developed to calculate the thermodynamic properties of such multi-component systems. In continuous thermodynamics, a distribution function replaces the discrete composition variable(usually the mole fraction) such that for a system of many components, calculation of thermodynamic properties is much simplified. A review concerning the application of continuous thermodynamics to the calculation of phase equilibria was given by Cotterman [7].

\footnotetext{
${ }^{*}$ To whom correspondence should be addressed
} 
Thermodynamic stability theory can be used to calculate the critical properties of a fluid mixture [8,9,11-15]. A continuous "component" comprises an infinite number of true components. Therefore, for a continuous "component, it is impractical to use the determinantform stability criteria for mixtures of discrete components where the dimension of the determinant is equal to the number of true components.

Johnson et. al. [8] established stability criteria for a polydisperse system; they showed that these criteria are equivalent to those for a system containing an infinite number of discrete components. These criteria can be expressed as a Fredholm determinant in the form of an infinite series. But the method of Johnson et. al. is not practical because of the infinite series; reduction to practice requires truncating the infinite series[9], thereby introducing error. However, for some systems, such as paraffin mixtures and polydisperse polymer solutions, the parameters of an equation of state can be expressed by simple functions of a suitable characterizing variable, such as molecular weight, boiling point, the size of particles etc. For example, Cotterman et. al. [10] found that when the Lorentz-Berthelot mixing rules are used in the Soave-Redlich-Kwong(SRK) equation of state, the square root of the attractive parameter $\left(a^{1 / 2}\right)$ and the repulsive parameter $(b)$ are linear functions of alkane molecular weight. Thus, the parameters of the SRK equation of state can be expressed as functions of mean molecular weight, or, more general, by the moments of the characterizing variable of the distribution function. In such cases, the stability criteria can be much simplified. They can be expressed by a function of the moments of the characterizing variable, e.g. by average molecular weight which is the first moment of the molecular-weight distribution.

Browarzik et. al.[11,12], $\mathrm{Hu}$ et. al.[13,14] and Cai et. al.[15] derived stability criteria for a polydisperse system described by an equation of state whose parameters are linear functions of average molecular weight $(\bar{M})$. Bellier-Castella et. al. [16] derived stability criteria for a polydisperse system modeled by the van der Waals equation of state where the parameters are functions of the average square and the average cube of the molecular $\operatorname{diameter}\left(\overline{\sigma^{2}}, \overline{\sigma^{3}}\right)$. However, because these earlier studies considered only some specific moments of the distribution variables, the critical criteria are not general. For example, the criteria for a free-energy model that is a function of the first-order moment are different from those for the same free energy model that is a function of higher-order moments. Although previous authors[11-16] claimed that their methods can be used to derive the stability criteria when the free energy is a function of any 
order moments, it is difficult (or at least very tedious) to use their method to derive a more general theory. Unfortunately, the most widely used free-energy models (or equations of state) of polydisperse systems are usually concerned with higher-order moments. For example, the hardsphere contribution to the free energy in the SAFT model is a function of the average diameter, the average square diameter and the average segment volume [17]. At this time, when applied to continuous systems we do not know the stability criteria for those models which depend on higher-order moments of model parameters.

This work provides a method for deriving the critical criteria of polydisperse systems where the residual part of the free energy is a function of the moments of one of more distribution variables. In this work, we derive the criteria for the critical point of a system containing one discrete and one continuous "component". Our focus is directed to a method that uses arbitrary moments of the distribution variables for establishing critical criteria. Although our work is applicable to systems characterized by more than one continuous variable, we confine attention to a system in which there is only one distribution function of a single variable. Our derivation is inspired by Heideman's work [18] concerning critical criteria for a multicomponent system described by discrete compositions. Following our derivation, we calculate the critical loci of alkane mixtures in which methane is a discrete component and all other alkanes comprise a continuous "component". Such mixtures are encountered in natural-gas technology.

\section{Critical criteria of a system that contains one discrete and one continuous "component"}

Consider a system containing one discrete and one continuous (polydisperse) "component". The free energy per unit volume $f$ is given by

$$
f=f^{\mathrm{id}}+f^{\mathrm{r}}=\rho_{\mathrm{s}}\left(A_{\mathrm{s}}^{0}+R T \ln \rho_{\mathrm{s}}\right)+\sum_{i=1}^{K} \rho_{i}\left[A_{i}^{0}+R T \ln \rho_{i}\right]+f^{\mathrm{r}}
$$

where $f^{\mathrm{id}}, f^{\mathrm{r}}$ and $A_{i}^{0}$ are, respectively, contributions from the ideal-gas mixture, from the residual part of the Helmhotz energy per unit volume and from the molar standard-state Helmholtz energy of virtual component $i$ in the continuous "component"; $\rho_{i}(i=1, \cdots K)$ is the molar density of virtual component $i$ in the continuous component where $K$ is a very large number; $\rho_{\mathrm{s}}$ is the molar density of the discrete component (e.g. methane); $R$ is the gas constant and $T$ is temperature. The residual part of the Helmholtz energy is a function of the parameters of the model used to describe the system. For example, for the van der Waals equation, parameter 
$a$ is a measure of attractive forces and parameter $b$ is a measure of repulsive forces. For the square-well fluid, the parameters are particle size and depth and width of the square well. For a continuous component, it is convenient to express the residual part of the Helmholtz energy as a function of the moments, i.e.

$$
f^{\mathrm{r}}=f^{\mathrm{r}}\left(T, \rho_{\mathrm{s}}, \rho_{\mathrm{t}}, \rho_{\mathrm{t}} \bar{M}, \rho_{\mathrm{t}} \overline{M^{2}}, \rho_{\mathrm{t}} \overline{M^{3}}, \cdots, \rho_{\mathrm{t}} \overline{M^{N}}\right)
$$

where $M$ is the selected characterizing variable of the distribution function of the continuous component, e.g. molecular weight; $\overline{M^{m}}$ is the $m$-th order moment; $N$ is the order of the highest order moment involved in the model; $\rho_{t}$ is the total molar density of the continuous component. In the continuous-component limit, i.e. $K \rightarrow \infty$, the density distribution can be described by a distribution function $\rho_{\mathrm{t}} \cdot \varphi(M)$ where $\varphi(M)$ is a probability distribution function with normalization

$$
\int \varphi(M) \mathrm{d} M=1
$$

The moments are defined by

$$
\overline{M^{m}}=\rho_{\mathrm{t}}^{-1} \sum_{i=1}^{K} \rho_{i} M_{i}^{m}=\int M^{m} \varphi(M) \mathrm{d} M
$$

where $M_{i}$ represents the distribution variable $M$ of the $i$-th virtual discrete component in the continuous "component". The $2^{\text {nd }}$-order variation of Eq (1) is given by

$$
\begin{aligned}
\delta^{2} f & =\frac{R T}{\rho_{\mathrm{s}}}\left(\delta \rho_{\mathrm{s}}\right)^{2}+\sum_{i=1}^{K} \frac{R T}{\rho_{i}}\left(\delta \rho_{i}\right)^{2}+\frac{\partial^{2} f^{\mathrm{r}}}{\partial \rho_{\mathrm{s}}^{2}}\left(\delta \rho_{\mathrm{s}}\right)^{2}+2 \sum_{m=0}^{N} \frac{\partial^{2} f^{\mathrm{r}}}{\partial \rho_{\mathrm{s}} \partial \rho_{\mathrm{t}} \overline{M^{m}}} \delta \rho_{\mathrm{s}} \delta \rho_{\mathrm{t}} \overline{M^{m}} \\
& +\sum_{l, m=0}^{N} \frac{\partial^{2} f^{\mathrm{r}}}{\partial \rho_{\mathrm{t}} \overline{M^{l}} \partial \rho_{\mathrm{t}} \overline{M^{m}}} \delta \rho_{\mathrm{t}} \overline{M^{l}} \delta \rho_{\mathrm{t}} \overline{M^{m}}
\end{aligned}
$$

To derive stability criteria, the key problem concerns the second term on the right hand side of Eq. (5). This term contains an infinite number of variations $\delta \rho_{i}$. The detailed derivation is given in the Appendix. Here we only give the final result.

\subsection{Spinodal Criterion}

The spinodal criterion is given by function $F_{\mathrm{sp}}$ :

$$
F_{\text {sp }}=\operatorname{det} \mathbf{A}_{(N+2) \times(N+2)}=0
$$

where subscript sp represents "spinodal" and the matrix $\mathbf{A}$ is given by 


$$
\mathbf{A}_{(N+2) \times(N+2)}=\left(\begin{array}{ccccc}
j_{\mathrm{s}}+f_{\mathrm{ss}}^{\mathrm{r}} & f_{\mathrm{s} 0}^{\mathrm{r}} & f_{\mathrm{s} 1}^{\mathrm{r}} & \cdots & f_{\mathrm{s} N}^{\mathrm{r}} \\
\sum_{m=0}^{N} f_{\mathrm{s} m}^{\mathrm{r}} \overline{M^{m}} & j_{\mathrm{t}}+\sum_{m=0}^{N} f_{m 0}^{\mathrm{r}} \overline{M^{m}} & \sum_{m=0}^{N} f_{m 1}^{\mathrm{r}} \overline{M^{m}} & \cdots & \sum_{m=0}^{N} f_{m N}^{\mathrm{r}} \overline{M^{m}} \\
\sum_{m=0}^{N} f_{\mathrm{s} m}^{\mathrm{r}} \overline{M^{m+1}} & \sum_{m=0}^{N} f_{m 0}^{\mathrm{r}} \overline{M^{m+1}} & j_{\mathrm{t}}+\sum_{m=0}^{N} f_{m 1}^{\mathrm{r}} \overline{M^{m+1}} & \cdots & \sum_{m=0}^{N} f_{m N}^{\mathrm{r}} \overline{M^{m+1}} \\
\vdots & \vdots & \vdots & \ddots & \vdots \\
\sum_{m=0}^{N} f_{\mathrm{s} m}^{\mathrm{r}} \overline{M^{m+N}} & \sum_{m=0}^{N} f_{m 0}^{\mathrm{r}} \overline{M^{m+N}} & \sum_{m=0}^{N} f_{m 1}^{\mathrm{r}} \overline{M^{m+N}} & \cdots & j_{\mathrm{t}}+\sum_{m=0}^{N} f_{m N}^{\mathrm{r}} \overline{M^{m+N}}
\end{array}\right)
$$

where $j_{\mathrm{s}}=R T \rho_{\mathrm{s}}^{-1}, j_{\mathrm{t}}=R T \rho_{\mathrm{t}}^{-1}$; subscript $(N+2) \times(N+2)$ represents the $N+2$ columns and rows of matrix $\mathbf{A}$; subscripts $s, m$ represent the derivative with respect to $\rho_{\mathrm{s}}$ and with respect to $\rho_{\mathrm{t}} \overline{M^{m}}, m=0,1, \cdots, N$, respectively.

\subsection{Critical Criterion}

The critical criterion is given by function $F_{\mathrm{cr}}$ :

$$
F_{\text {cr }}=\left|\begin{array}{cccc}
A_{11} & A_{12} & \cdots & A_{1,2 N+2} \\
A_{31}-\bar{M} A_{21} & A_{32}-\bar{M} A_{22} & \cdots & A_{3,2 N+2}-\bar{M} A_{3,2 N+2} \\
\vdots & \vdots & \ddots & \vdots \\
A_{2 N+2,1} & A_{2 N+2,2} & \cdots & A_{2 N+2,2 N+2} \\
\frac{\partial F_{\mathrm{sp}}}{\partial \rho_{\mathrm{s}}} & \frac{\partial F_{\mathrm{sp}}}{\partial \rho_{\mathrm{t}}} & \cdots & \frac{\partial F_{\mathrm{sp}}}{\partial \rho_{\mathrm{t}} \overline{M^{2 N}}}
\end{array}\right|=0
$$

where subscript cr represents "critical" and $A_{i j}$ is the element of matrix $\mathbf{A}$.

\section{Stability test}

To locate critical points, two stability criteria must also be satisfied [18]; they are

(1) Mechanical stability:

$$
p>0, \quad\left(\frac{\partial p}{\partial V}\right)_{T} \geq 0 \text { and }
$$

(2) Phase splitting stability:

$$
\begin{aligned}
& \left.\left(1-x_{\mathrm{t}}\right) \mid \mu_{\mathrm{s}}\left(T^{\mathrm{c}}, p^{\mathrm{c}}, x_{\mathrm{t}} ;[\varphi]\right)-\mu_{\mathrm{s}}^{\mathrm{c}}\left(T^{\mathrm{c}}, p^{\mathrm{c}}, x_{\mathrm{t}}^{\mathrm{c}} ;\left[\varphi^{\mathrm{c}}\right]\right)\right] \\
& +x_{\mathrm{t}} \int \varphi(M)\left[\mu_{M}\left(T^{\mathrm{c}}, p^{\mathrm{c}}, x_{\mathrm{t}} ;[\varphi]\right)-\mu_{M}^{\mathrm{c}}\left(T^{\mathrm{c}}, p^{\mathrm{c}}, x_{\mathrm{t}}^{\mathrm{c}} ;\left[\varphi^{\mathrm{c}}\right]\right)\right] \mathrm{d} M>0
\end{aligned}
$$


where $T^{\mathrm{c}}$ and $p^{\mathrm{c}}$ are the roots of Eqs.(6) and (8), $\mu_{\mathrm{s}}$ and $\mu_{M}$ are, respectively, the chemical potential of the discrete component and that of the continuous "component" with variable $M ; x_{\mathrm{t}}$ is the mole fraction of the continuous "component"; superscript c represents the system of interest whose critical temperature and pressure is calculated. Eq. (10) requires that we must try various $x_{\mathrm{t}}$ and various forms of distribution function $\varphi(M)$ to calculate the chemical potential and compare them with the one of interest $\left(x_{\mathrm{t}}^{\mathrm{c}}, \varphi^{\mathrm{c}}(M)\right)$. The mole fraction $x_{\mathrm{t}}$ and distribution function $\varphi(M)$ can be of any form that satisfies the conditions $0<x_{\mathrm{t}}<1$ and $\int \varphi(M) \mathrm{d} M=1$ and $\varphi(M) \geq 0$. If there exists a value of $x_{\mathrm{t}}$ or a form of $\varphi(M)$ such that Eq.(10) is not satisfied while eqs.(9) are valid, then roots $T^{\mathrm{c}}$ and $p^{\mathrm{c}}$ of Eqs. (6) and (8) are not the correct critical point because they probably describe a metastable state. It is impractical to calculate Eq. (10) with all possible forms of $\varphi(u)$. Fortunately, Bellier-Castella et. al. [16] have shown that we need not try various distribution functions. We only need to calculate Eq. (10) with various values of $x_{t}$. For a continuous "component", the chemical potential is defined by a functional derivative given by [6]:

$$
\mu_{M}=\frac{\delta f}{\delta \rho_{t} \varphi(M)}
$$

The free energy per unit volume can be expressed as a functional of the distribution function

$$
\begin{aligned}
f & =\rho_{\mathrm{s}} A_{\mathrm{s}}^{0}+\rho_{\mathrm{s}} k T \ln \left(\rho_{\mathrm{s}} R T / p^{0}\right)+\int \rho_{\mathrm{t}} \varphi(M)\left[A^{0}(M)+k T \ln \left(\rho_{\mathrm{t}} R T \varphi(M) \Delta M / p^{0}\right)\right] \mathrm{d} M \\
& +f^{\mathrm{r}}\left(T, \rho_{\mathrm{s}} ;\left[\rho_{\mathrm{t}} \varphi\right]\right)
\end{aligned}
$$

where $\left[\rho_{\mathrm{t}} \varphi\right]$ means functional and $\Delta M$ is a constant which does not affect the result. Substituting Eq. (12) into Eq.(11), the chemical potential of a continuous component is given by

$$
\mu(M)=A^{0}(M)+R T+R T \ln \frac{\rho_{\mathrm{t}} R T \varphi(M) \Delta M}{p^{0}}+\frac{\delta f^{\mathrm{r}}}{\delta \rho_{\mathrm{t}} \varphi(M)}
$$

Because the residual part of the free energy is only a function of moments(see $\mathrm{Eq}(2))$, the functional derivative can be expressed as

$$
\frac{\delta f^{\mathrm{r}}}{\delta \rho_{\mathrm{t}} \varphi(M)}=\frac{\partial f^{\mathrm{r}}}{\partial \rho_{\mathrm{t}}} \frac{\delta \rho_{\mathrm{t}}}{\delta \rho_{\mathrm{t}} \varphi(M)}+\sum_{i=1}^{N} \frac{\partial f^{\mathrm{r}}}{\partial \overline{M^{i}}} \frac{\delta \overline{M^{i}}}{\delta \rho_{\mathrm{t}} \varphi(M)}
$$

where the functional derivatives are given by 


$$
\begin{aligned}
& \frac{\delta \rho_{\mathrm{t}}}{\delta \rho_{\mathrm{t}} \varphi(M)}=1 \\
& \frac{\delta \overline{M^{i}}}{\delta \rho_{\mathrm{t}} \varphi(M)}=\frac{M^{i}-\overline{M^{i}}}{\rho_{\mathrm{t}}}
\end{aligned}
$$

The chemical potential of the discrete component is obtained from the customary thermodynamic relation

$$
\mu_{\mathrm{s}}=A_{\mathrm{s}}^{0}+R T \ln \frac{\rho_{\mathrm{s}} R T}{p^{0}}+\frac{\partial f^{\mathrm{r}}}{\partial \rho_{\mathrm{s}}}
$$

\section{Reduction to Practice: the SRK Equation of State}

To illustrate the stability criteria developed in this work, we calculate the critical loci of alkane mixtures where methane is the discrete component and all other alkanes form a continuous pseudo-component. We use Soave's modified Redlich-Kwong(SRK) equation of state:

$$
p=\frac{R T}{v-b}-\frac{a(T)}{v(v+b)}=\frac{\rho R T}{1-\rho b}-\frac{\rho^{2} a(T)}{1+\rho b}
$$

where $p$ is the pressure; $T$ is the temperature; $v$ is the molar volume; $\rho=v^{-1}$ is the molar density and $a(T), b$ are the equation-of-state parameters. Pure-component parameters are obtained from pure-component critical temperatures and pressures and from Pitzer's acentric factor $\omega$ :

$$
\begin{aligned}
& a(T)=0.4275 \frac{R^{2} T_{\mathrm{c}}^{2}}{p_{\mathrm{c}}} \alpha(T) \\
& \alpha(T)=\left[1+m\left(1-\sqrt{T / T_{\mathrm{c}}}\right)\right]^{2} \\
& m=0.48+1.57 \omega-0.176 \omega^{2} \\
& b=0.08664 \frac{R T_{\mathrm{c}}}{p_{\mathrm{c}}}
\end{aligned}
$$

For mixtures, the mixing rules chosen here are

$$
\begin{aligned}
& a(T)=\sum_{i, j=1}^{K} x_{i} x_{j} a_{i j}(T), \quad a_{i j}(T)=\sqrt{a_{i}(T) a_{j}(T)}\left(1-k_{i j}\right) \\
& b=\sum_{i=1}^{K} x_{i} b_{i}
\end{aligned}
$$


where $k_{i j}$ is a binary interaction parameter $\left(k_{i i}=k_{j j}=0\right)$ and $x_{i}$ is mole fraction. From the SRK equation, the residual part of the Helmholtz energy per unit volume is

$$
f^{\mathrm{r}}\left(T, \rho_{1}, \ldots, \rho_{K}\right)=-\rho R T \ln (1-\rho b)-\rho a b^{-1} \ln (1+\rho b)
$$

where $\rho=\sum_{i} \rho_{i}$ is the total molar density. For a system containing one discrete component and one continuous component, the ideal part of the Helmholtz energy per unit volume is given by

$$
\begin{aligned}
& f^{\text {id }}=\rho_{\mathrm{s}} A_{\mathrm{s}}^{0}+\rho_{\mathrm{s}} R T \ln \left(\rho_{\mathrm{s}} R T / p^{0}\right)+\int \mathrm{d} M \cdot \rho_{\mathrm{t}} \varphi(M)\left[A^{0}(M)+k T \ln \left(\rho_{\mathrm{t}} R T \varphi(M) \Delta M / p^{0}\right)\right] \\
& f^{\mathrm{r}}=-\rho R T \ln (1-\rho b)-\rho a(T) b^{-1} \ln (1+\rho b)
\end{aligned}
$$

where $\rho=\rho_{\mathrm{t}}+\rho_{\mathrm{s}}$ is the total molar density of the mixture and where $a(T)$ and $b$ are given by

$$
\begin{aligned}
& \rho^{2} a(T)=a_{\mathrm{s}}(T) \rho_{\mathrm{s}}^{2}+2 \rho_{\mathrm{s}} \rho_{\mathrm{t}} \int \varphi(M) \sqrt{a(M, T) a_{\mathrm{s}}(T)}\left(1-k_{\mathrm{s} M}\right) \mathrm{d} M \\
& \quad+\rho_{\mathrm{t}}^{2} \iint \varphi(M) \varphi\left(M^{\prime}\right) \sqrt{a(M, T) a\left(M^{\prime}, T\right)}\left(1-k_{M M^{\prime}}\right) \mathrm{d} M \mathrm{~d} M^{\prime} \\
& \rho b=\rho_{\mathrm{s}} b_{\mathrm{s}}+\rho_{\mathrm{t}} \int \varphi(M) b(M) \mathrm{d} M
\end{aligned}
$$

Cotterman et. al. [10] showed that for the SRK equation applied to paraffins, $\sqrt{a(T)}$ and $b$ are linear functions of molecular weight $M$

$$
\begin{aligned}
& \sqrt{a(M, T)}=a^{(0)}(T)+a^{(1)}(T) M \\
& b(M)=b^{(0)}+b^{(1)} M
\end{aligned}
$$

where $a^{(0)}, a^{(1)}, b^{(0)}$ and $b^{(1)}$ are known constants. Substituting Eq. (23) into Eq. (22), we obtain parameters $a, b$ for the mixture:

$$
\begin{aligned}
& \rho^{2} a(T)=a_{\mathrm{s}}(T) \rho_{\mathrm{s}}^{2}+2 \rho_{\mathrm{s}}\left[\rho_{\mathrm{t}} a^{(0)}(T)+a^{(1)}(T) \rho_{\mathrm{t}} \bar{M}\right] \sqrt{a_{\mathrm{s}}(T)}\left(1-k_{\mathrm{s} M}\right) \\
& +\left[\rho_{\mathrm{t}} a^{(0)}(T)+a^{(1)}(T) \rho_{\mathrm{t}} \bar{M}\right]^{2} \\
& \rho b=\rho_{\mathrm{s}} b_{\mathrm{s}}+\rho_{\mathrm{t}} b^{(0)}+b^{(1)} \rho_{\mathrm{t}} \bar{M}
\end{aligned}
$$

In Eq. (23) and (24) the binary interaction parameter $k_{i j}(i \neq j)$ is assumed to be 0 . These relations show that the Helmholtz energy is a function of temperature $T$, molar density of discrete component $\rho_{s}$, molar density of continuous component $\rho_{t}$ and the average molecular weight of the continuous "components" multiplied by its molar density: $\rho_{\mathrm{t}} \bar{M}$. For paraffins, Eqs (23) is given by 


$$
\begin{gathered}
\sqrt{a(M, T)}=\left(0.029938-2.8123 \times 10^{-5} T+8.4697 \times 10^{-9} T^{2}\right) M \\
+\left(-0.0022345+1.3978 \times 10^{-4} T-4.2098 \times 10^{-8} T^{2}\right) \\
b(M)=-0.015564+0.0016308 M
\end{gathered}
$$

where the unit of temperature is kelvins; the unit of molecular weight is $\mathrm{g} \cdot \mathrm{mol}^{-1}$; the unit of $a$ is $\mathrm{pa} \cdot \mathrm{m}^{6} \mathrm{~mol}^{-2}$; and the unit of $b$ is $\mathrm{m}^{3} \mathrm{~mol}^{-1}$. Critical temperatures, pressures and acentric factors for pure paraffins are from Reid et. al. [19]. Figs. 1a and 1b show the molecular-weight dependence of parameters $a$ and $b$ for normal alkanes from ethane to hexadecane.

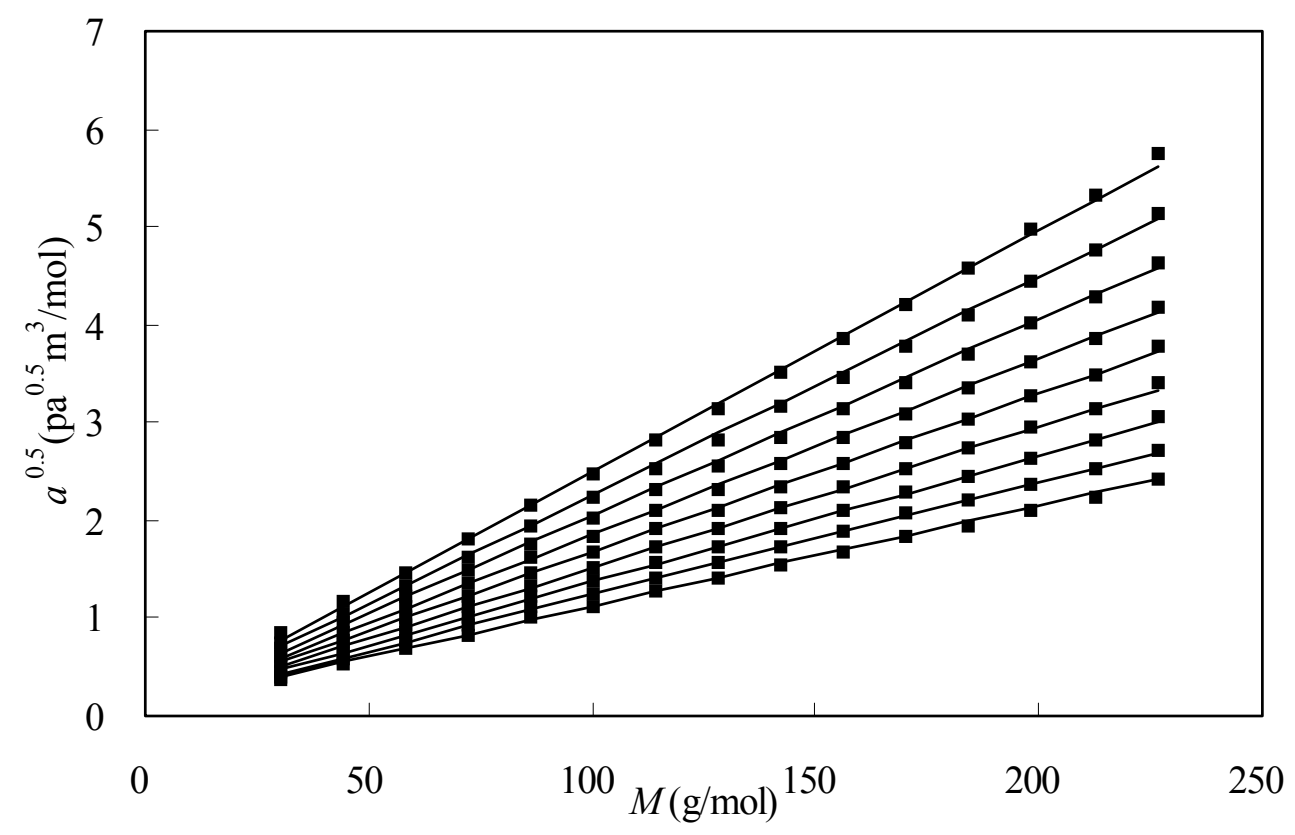

Fig.1a Molecular-weight and temperature dependence of SRK parameter $a(T)$.

Dots: from Eq.(18). Lines: from Eq. (25a)

From top to bottom, the temperature changes from 200 to $1000 \mathrm{~K}$ in intervals of $100 \mathrm{~K}$. 


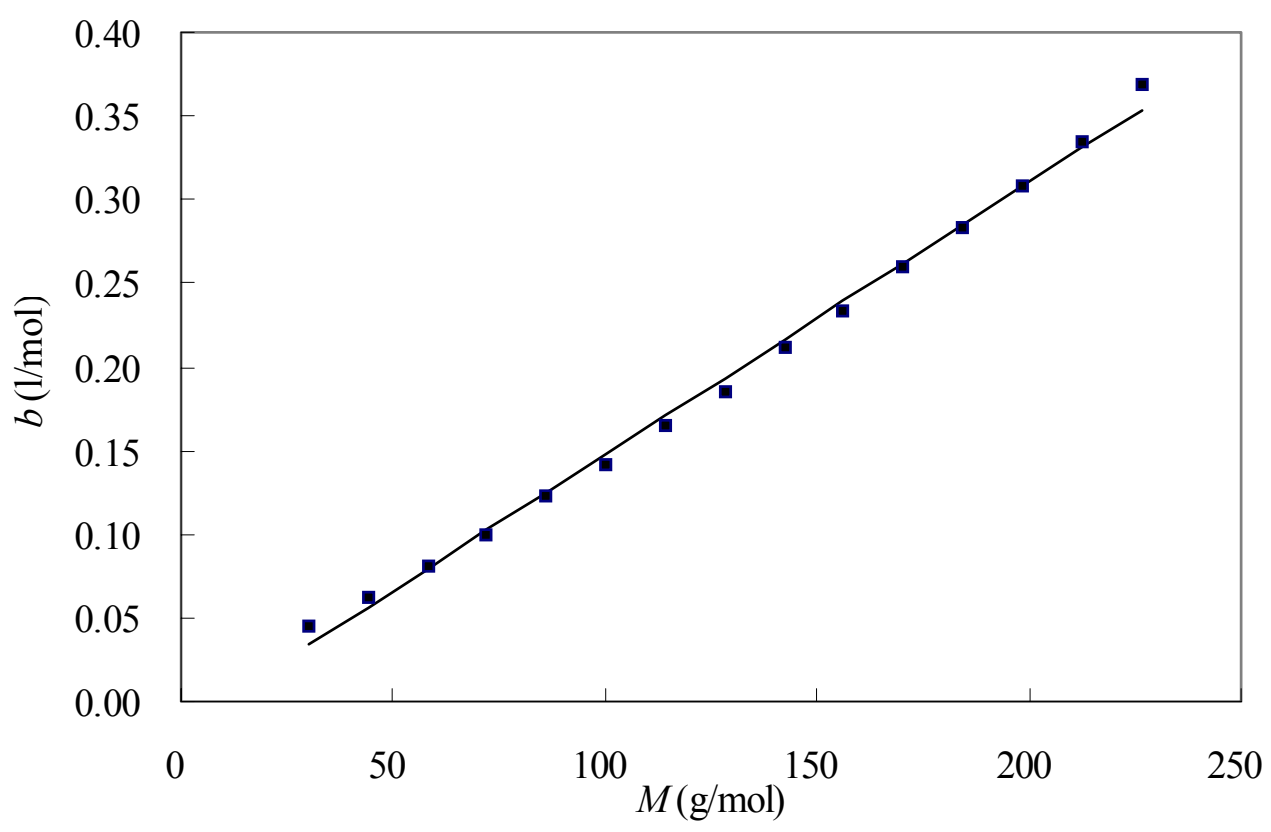

Fig. 1b Molecular-weight dependence of SRK parameter $b$.

Dots: from Eq.(18). Lines: from Eq. (25b)

\section{Illustrative Results for Alkane Mixtures}

We use the numerical algorithm based on a one-dimensional search suggested by Hicks and Young [20] for obtaining the roots of Eqs (6) and (8). Although this method requires many evaluations of objective functions Eqs. (6) and (8), it guarantees finding all the roots in a given region. The stability test is performed after obtaining the roots of Eqs.(6) and (8). In the figures to follow, we omit curves showing the limits of unstable and metastable regions.

The SRK equation of state gives good results for the critical loci of binary alkane mixtures. Here we give two examples. Fig. 2 shows the Type I critical locus for the binary system methanepropane. (For classification of critical loci, see the review by van Konynenburg and Scott [21] and the book by J. Rowlinson [22].) Experimental data [23] are in good agreement with calculated results using $k_{13}=0$. Fig. 3a shows the critical locus for the binary system methanehexane; Fig. $3 \mathrm{~b}$ gives a local enlargement of Fig. 3a near the critical point of methane. Calculations are based on $k_{16}=0$. Fig. 3 shows that the critical locus of methane-hexane is of Type V. In this case, there exists a three-phase liquid-liquid-gas equilibrium near the critical temperature and pressure of methane. At a certain pressure, there exist two critical points, one is 
a liquid-liquid critical point and the other is a vapor-liquid critical point. Calculated results are in good agreement with experimental data $[23,24,25]$ but the small three-phase-equilibrium region is represented only qualitatively.

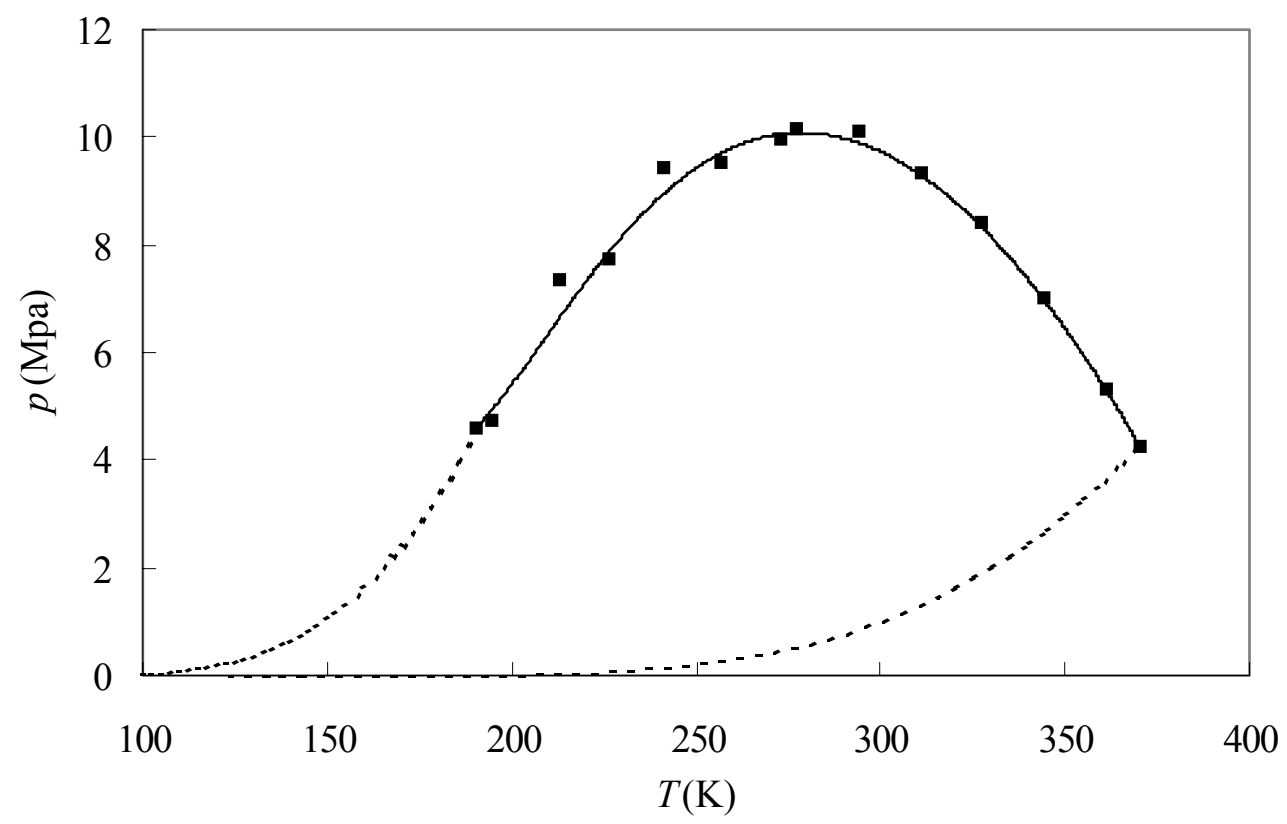

Fig. 2 Critical locus for the binary system methane-propane

Dots: experimental data [23]; line: calculated critical locus; dashed lines: saturated vapor pressures of the pure components 


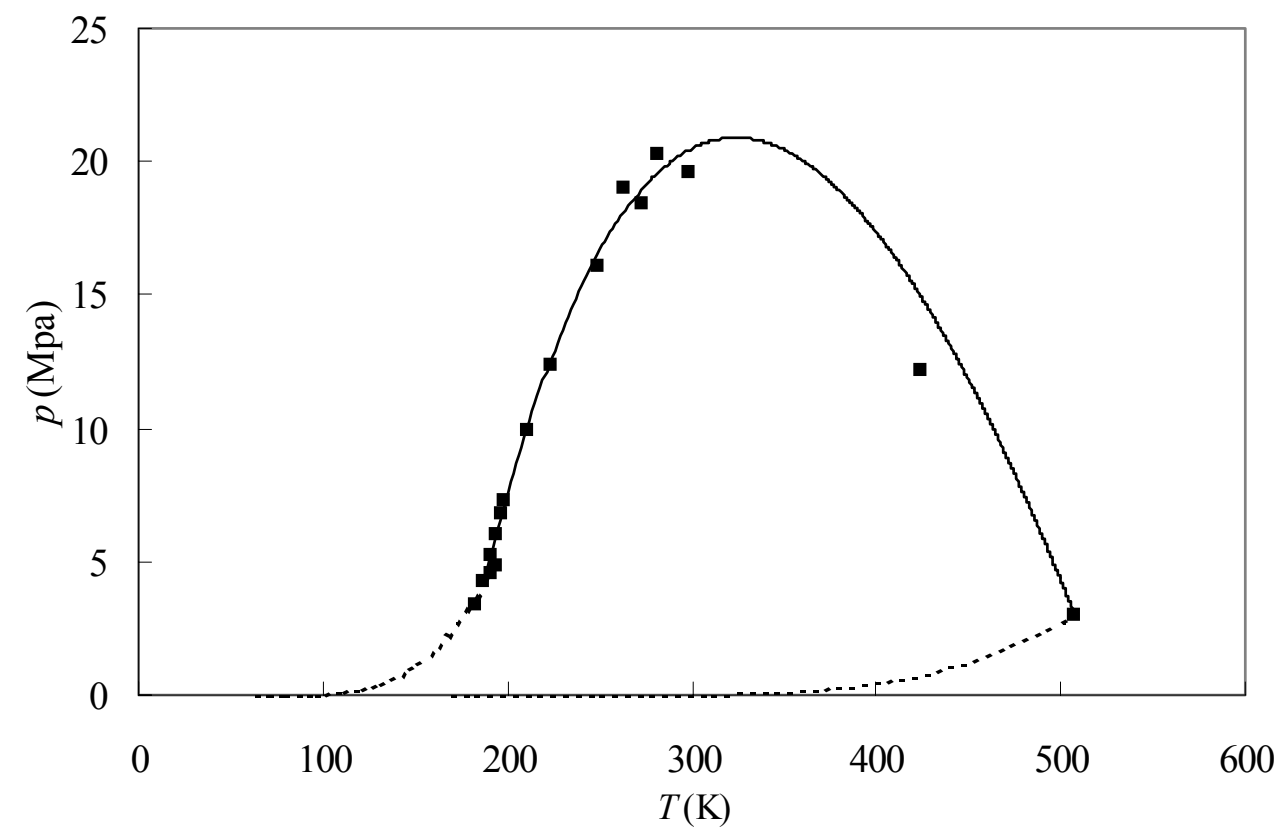

Fig. 3a Critical locus for the binary system methane-hexane

Dots: experimental data $[23,24,25]$; lines: calculated critical locus; dashed lines: saturated vapor pressures of the pure components

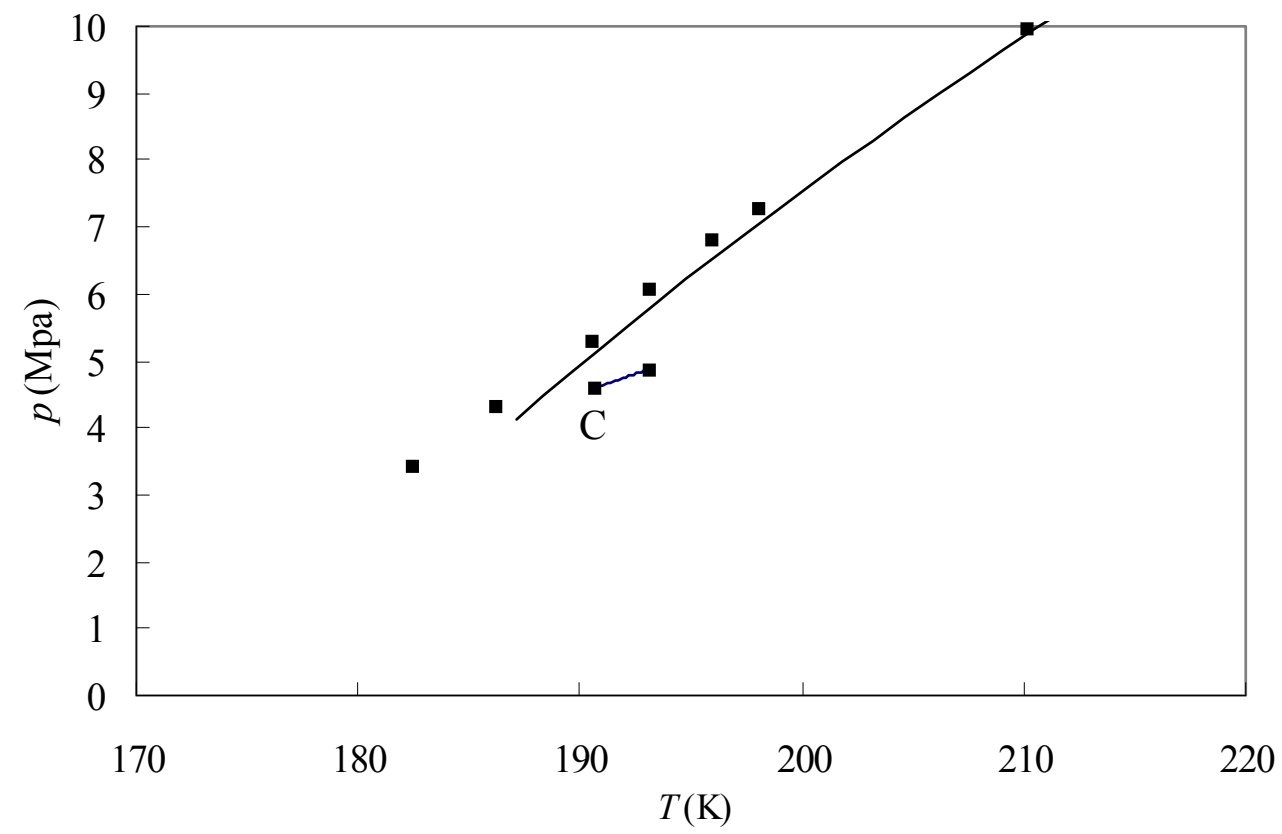

Fig. 3b A local Enlargement of Fig. 3a near the critical point of methane(C). The saturated vapor pressure of methane is omitted. 
We now consider a multicomponent mixture of normal alkanes. In a typical natural-gas mixtures, methane is present in excess. We choose methane as the discrete component and represent the concentration of ethane to hexadecane $\left(\mathrm{C}_{2}\right.$ to $\left.\mathrm{C}_{16}\right)$ by a beta-distribution function of the molecular weight:

$$
\varphi(M, \alpha, \beta)=\frac{1}{\left(M_{\max }-M_{\min }\right) \mathrm{B}(\alpha, \beta)}\left(\frac{M-M_{\min }}{M_{\text {max }}-M_{\text {min }}}\right)^{\alpha-1}\left(\frac{M_{\max }-M}{M_{\text {max }}-M_{\text {min }}}\right)^{\beta-1}
$$

where $M_{\min }$ and $M_{\max }$ are boundary values; $\mathrm{B}(\alpha, \beta)$ is the beta function:

$$
\mathrm{B}(\alpha, \beta)=\int_{0}^{1} t^{\alpha-1}(1-t)^{\beta-1} \mathrm{~d} t
$$

where $\alpha$ and $\beta$ are parameters determined by the average molar mass and the variance for the continuous "component". To include ethane and hexadecane, we choose for the lower boundary a molar mass slightly smaller than that of ethane and for the upper boundary, a molar mass slightly larger than that of hexadecane: we set $M_{\min }=25$ and $M_{\max }=230$.

Fig. 4a shows how the calculated critical locus depends on the mean molar mass while keeping the variance constant. Fig. $4 \mathrm{~b}$ is a local enlargement of Fig. 4a near the critical point of methane. When the mean molecular weight of the continuous "component" increases, the critical locus moves to higher temperatures. Fig. $4 \mathrm{~b}$ shows that, at a certain value of mean molar mass, the critical locus changes from Type I to Type V, i.e. from a continuous line to two discontinuous lines. Line 1 shows a Type I critical locus and line 2 shows a Type V critical locus. We do not find other types when the average molar mass lies between $M_{\min }$ and $M_{\max }$. 


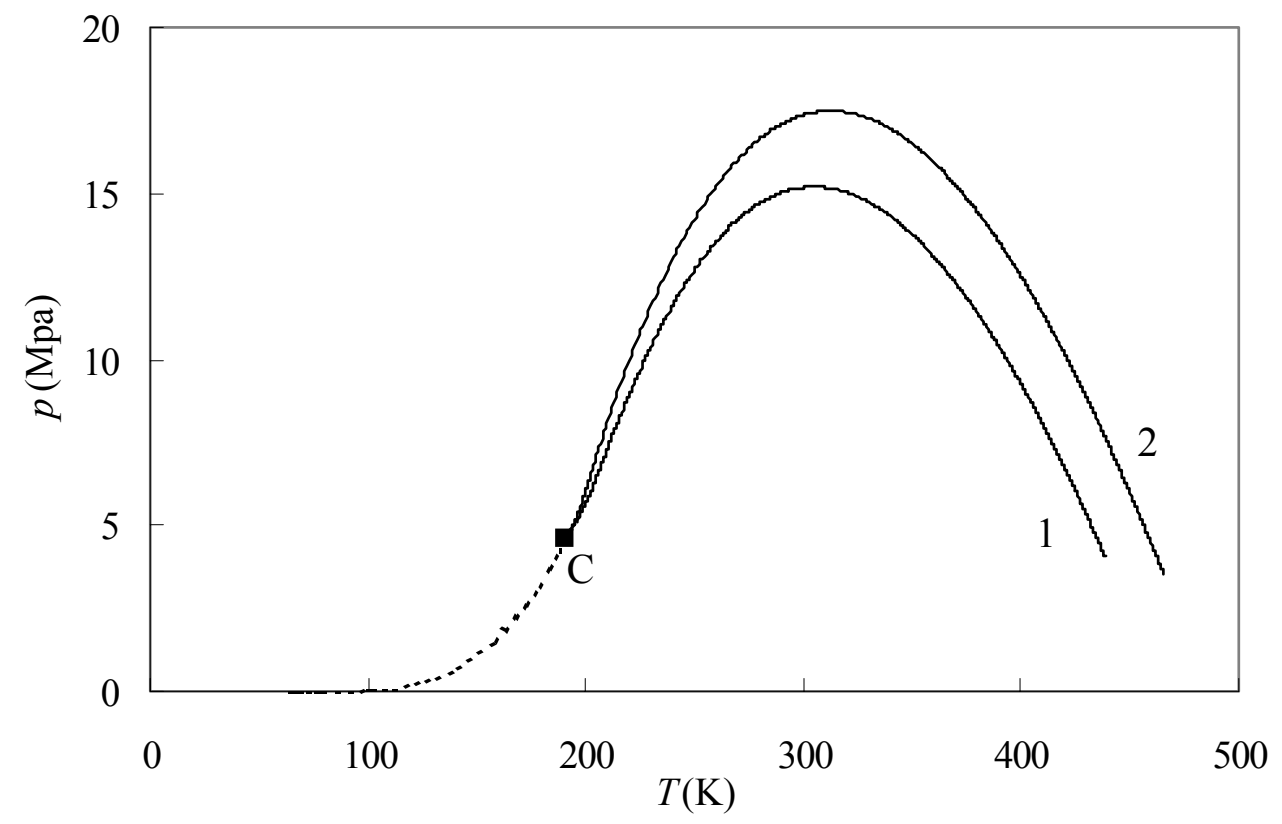

Fig. 4a Calculated critical locus for mixtures containing methane and a continuous alkane "component" from $\mathrm{C}_{2}$ to $\mathrm{C}_{16}$. Solid lines: critical loci; dashed line: saturated vapor pressures of methane; $\mathrm{C}$ is the critical point of methane; $1: \bar{M}=60, \bar{M}^{2}-\bar{M}^{2}=100 ; 2: \bar{M}=70$. For both cases 1 and 2, the variance $\overline{M^{2}}-\bar{M}^{2}=100$

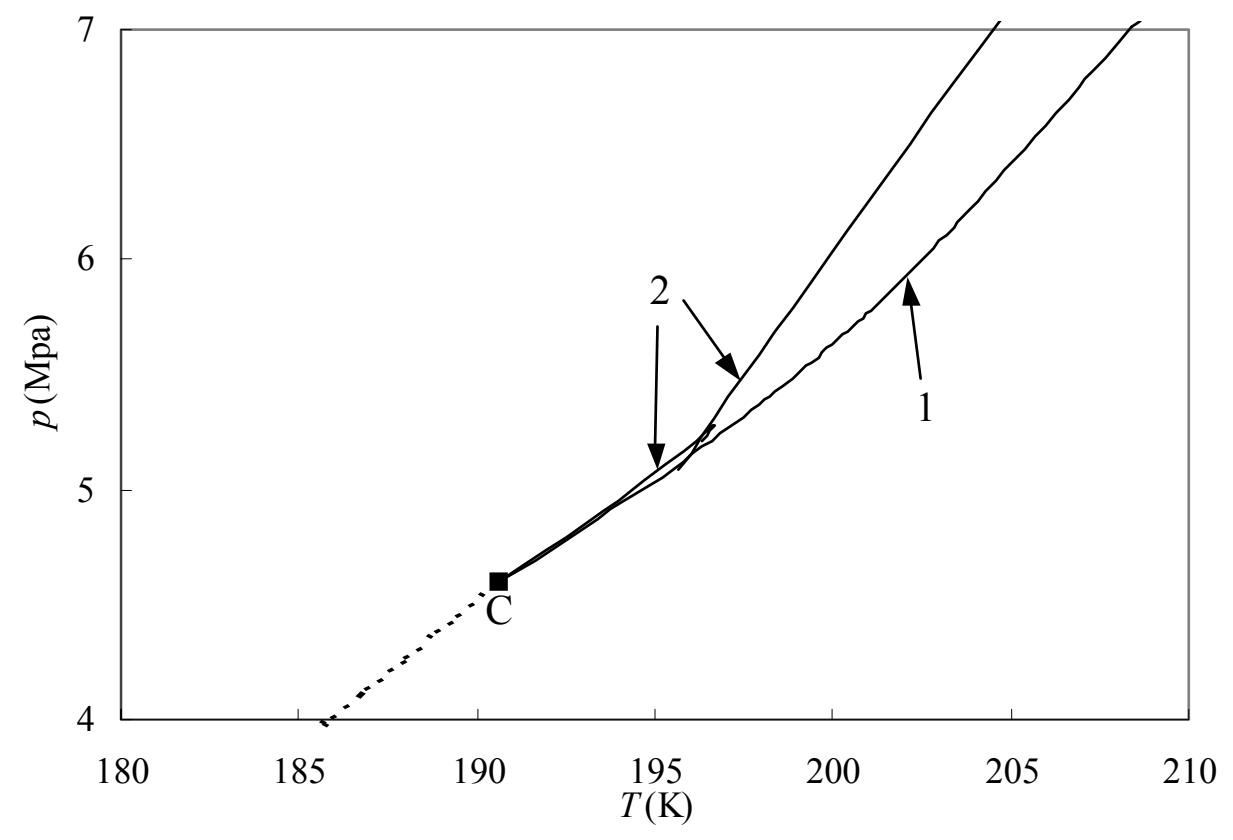

Fig. 4b A local enlargement of Fig. 4a 
Figs. $5 \mathrm{a}$ and $5 \mathrm{~b}$ show how the critical locus depends on the variance of the distribution function while keeping the mean molar mass constant. Fig. 5b is a local enlargement of Fig. 5a near the critical point of methane. When the variance increases, the critical locus extends to higher temperatures. Fig. 5b shows that at a certain value of the variance, the critical locus changes from Type I to Type V. Line 1 is a Type I critical locus and line 2 is a Type V critical locus.

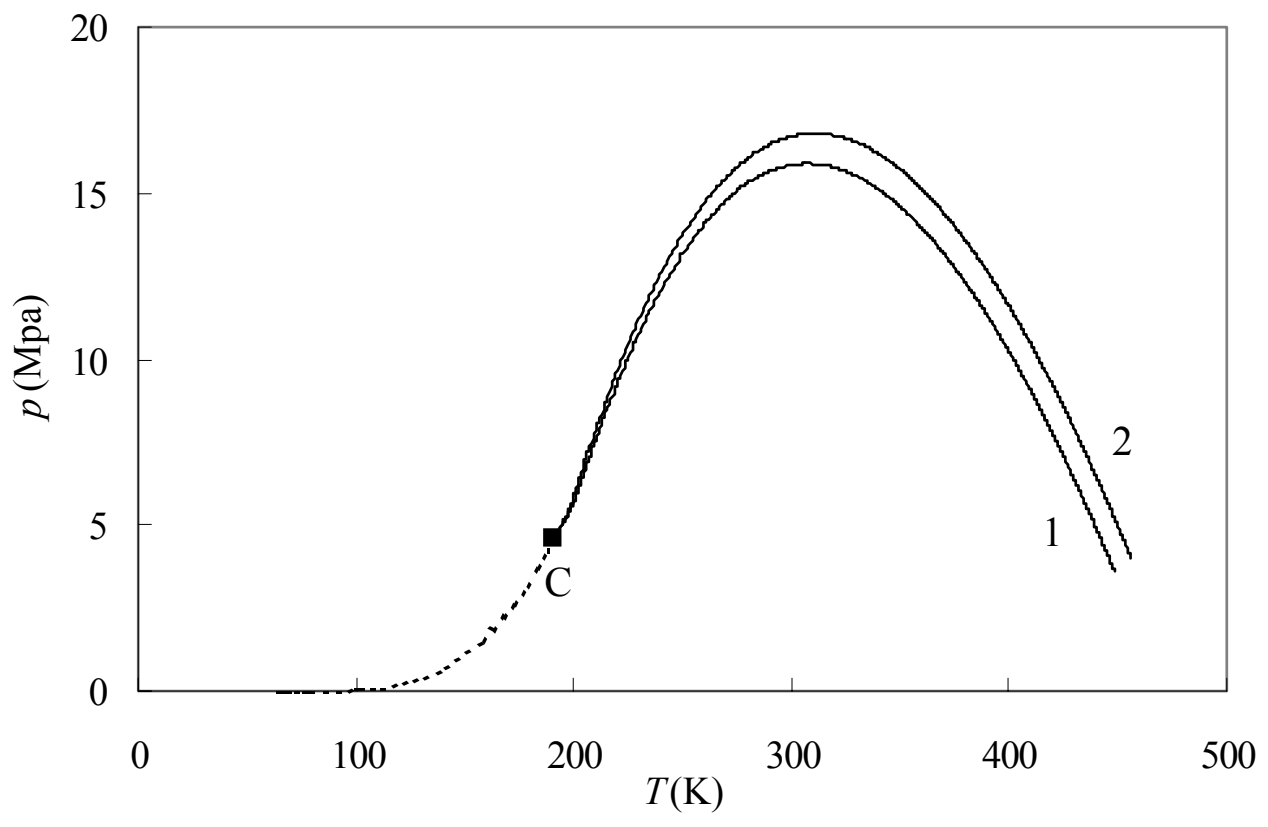

Fig. 5a Calculated critical locus for mixtures containing methane and a continuous alkane "component" from $\mathrm{C}_{2}$ to $\mathrm{C}_{16}$. Solid lines: critical loci; dashed line: saturated vapor pressure curve of methane; $\mathrm{C}$ is the critical point of methane; $1: \bar{M}^{2}-\bar{M}^{2}=10 ; 2: \bar{M}^{2}-\bar{M}^{2}=200$. For both lines 1 and 2, the mean molecular weight $\bar{M}=65$ 


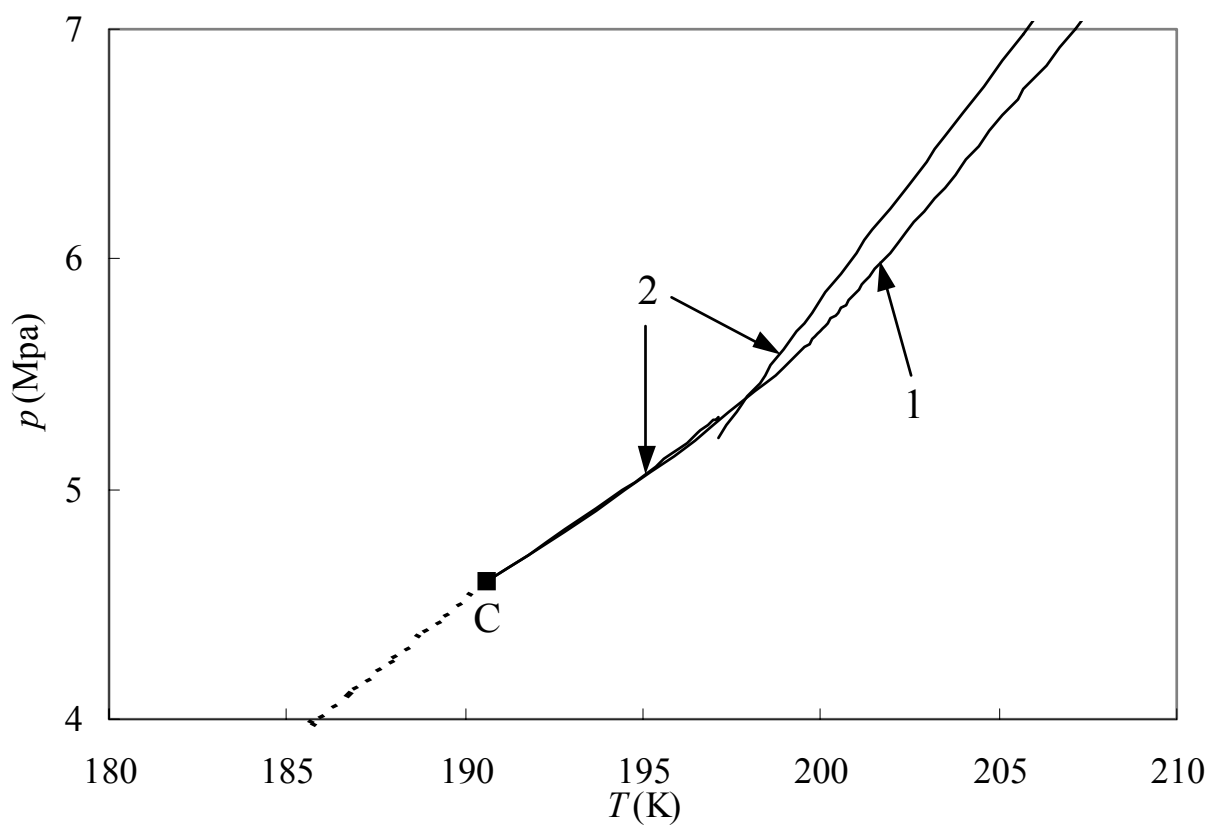

Fig. 5b A local enlargement of Fig. 5a

\section{Conclusion}

We have established stability criteria for a system containing one discrete and one continuous "component" where molecular-thermodynamic model parameters depend on the first and higherorder moments of the distribution variable. While the derivation described here can be generalized to systems that contain several discrete components and several continuous "components", the procedure for such systems is tedious (see Appendix).

To illustrate applicability, the SRK equation of state is used here for calculating the critical loci of natural-gas systems where (discrete) methane is in a (continuous) multicomponent mixture with higher normal alkanes up to hexadecane.

\section{Acknowledgement}

The authors are grateful to the Office for Basic Sciences, US Department of Energy, for financial support. Additional support was provided by the Energy Institute of the University of California and by the Chinese National Science Foundation. 


\section{Appendix :}

\section{Derivation of Spinodal Criterion}

According to stability theory [26], stability is attained when the $2^{\text {nd }}$-order variation of the free energy is non-negative, i.e.

$$
\delta^{2} f \geq 0 .
$$

where $f$ is the Helmholtz energy per unit volume. Here the molar densities are the independent variables. The $2^{\text {nd }}$-order variation of $\delta^{2} f$ is a quadratic form of the vector $\left(\delta \rho_{\mathrm{s}}, \delta \rho_{1}, \delta \rho_{2}, \cdots, \delta \rho_{K}\right)$; it is given by

$$
\delta^{2} f=\left(\delta \rho_{\mathrm{s}}, \delta \rho_{1}, \delta \rho_{2}, \cdots, \delta \rho_{K}\right)\left(\begin{array}{cccc}
\frac{\partial^{2} f}{\partial \rho_{\mathrm{s}}^{2}} & \frac{\partial^{2} f}{\partial \rho_{\mathrm{s}} \partial \rho_{1}} & \cdots & \frac{\partial^{2} f}{\partial \rho_{\mathrm{s}} \partial \rho_{K}} \\
\frac{\partial^{2} f}{\partial \rho_{\mathrm{s}} \partial \rho_{1}} & \frac{\partial^{2} f}{\partial \rho_{1}^{2}} & \cdots & \frac{\partial^{2} f}{\partial \rho_{1} \partial \rho_{K}} \\
\vdots & \vdots & \ddots & \vdots \\
\frac{\partial^{2} f}{\partial \rho_{\mathrm{s}} \partial \rho_{K}} & \frac{\partial^{2} f}{\partial \rho_{1} \partial \rho_{K}} & \cdots & \frac{\partial^{2} f}{\partial \rho_{K}^{2}}
\end{array}\right)\left(\begin{array}{c}
\delta \rho_{\mathrm{s}} \\
\delta \rho_{1} \\
\delta \rho_{2} \\
\vdots \\
\delta \rho_{K}
\end{array}\right) \geq 0 .
$$

To make Eq. (A2) larger than or equal to zero, the sufficient and necessary condition is that the determinant of the coefficient matrix is positive definite. In the limit of stability, the determinant vanishes. We obtain the well-known spinodal criterion for multicomponent system first suggested by Gibbs

$$
\left|\begin{array}{cccc}
\frac{\partial^{2} f}{\partial \rho_{\mathrm{s}}^{2}} & \frac{\partial^{2} f}{\partial \rho_{\mathrm{s}} \partial \rho_{1}} & \cdots & \frac{\partial^{2} f}{\partial \rho_{\mathrm{s}} \partial \rho_{K}} \\
\frac{\partial^{2} f}{\partial \rho_{\mathrm{s}} \partial \rho_{1}} & \frac{\partial^{2} f}{\partial \rho_{1}^{2}} & \cdots & \frac{\partial^{2} f}{\partial \rho_{1} \partial \rho_{K}} \\
\vdots & \vdots & \ddots & \vdots \\
\frac{\partial^{2} f}{\partial \rho_{\mathrm{s}} \partial \rho_{K}} & \frac{\partial^{2} f}{\partial \rho_{1} \partial \rho_{K}} & \cdots & \frac{\partial^{2} f}{\partial \rho_{K}^{2}}
\end{array}\right|=0 .
$$

Now we use Eq. (A2) to obtain the spinodal criterion for the system where one component is discrete and the other "component" is characterized by a distribution function. The objective now is to replace the derivatives of the free energy with respect to the density of a virtual component in Eq. (A3) by those with respect to moments of the distribution function. Eq. (A3) can be rewritten in the equivalent form 


$$
\left(\begin{array}{cccc}
\frac{\partial^{2} f}{\partial \rho_{\mathrm{s}}^{2}} & \frac{\partial^{2} f}{\partial \rho_{\mathrm{s}} \partial \rho_{1}} & \cdots & \frac{\partial^{2} f}{\partial \rho_{\mathrm{s}} \partial \rho_{K}} \\
\frac{\partial^{2} f}{\partial \rho_{\mathrm{s}} \partial \rho_{1}} & \frac{\partial^{2} f}{\partial \rho_{1}^{2}} & \cdots & \frac{\partial^{2} f}{\partial \rho_{1} \partial \rho_{K}} \\
\vdots & \vdots & \ddots & \vdots \\
\frac{\partial^{2} f}{\partial \rho_{\mathrm{s}} \partial \rho_{K}} & \frac{\partial^{2} f}{\partial \rho_{1} \partial \rho_{K}} & \cdots & \frac{\partial^{2} f}{\partial \rho_{K}^{2}}
\end{array}\right)\left(\begin{array}{c}
\delta \rho_{\mathrm{s}} \\
\delta \rho_{1} \\
\delta \rho_{2} \\
\vdots \\
\delta \rho_{K}
\end{array}\right)=\left(\begin{array}{c}
0 \\
0 \\
0 \\
\vdots \\
0
\end{array}\right) .
$$

Eq. (A3) is the sufficient and necessary condition that Eq. (A4) has a nontrivial solution. Using Eq. (1-4), the first linear equation in Eq. (A4) can be rewritten as

$$
\frac{R T}{\rho_{\mathrm{s}}} \delta \rho_{\mathrm{s}}+f_{\mathrm{ss}}^{\mathrm{r}} \delta \rho_{\mathrm{s}}+\sum_{k=1}^{K} \sum_{m=0}^{N} f_{\mathrm{s} m}^{\mathrm{r}} M_{k}^{m} \delta \rho_{k}=\frac{R T}{\rho_{\mathrm{s}}} \delta \rho_{\mathrm{s}}+f_{\mathrm{ss}}^{\mathrm{r}} \delta \rho_{\mathrm{s}}+\sum_{m=0}^{N} f_{\mathrm{s} m}^{\mathrm{r}} \delta \rho_{\mathrm{t}} \overline{M^{m}}=0
$$

where subscripts s, $m$ represent the derivative with respect to $\rho_{\mathrm{s}}$ and $\rho_{\mathrm{t}} \overline{M^{m}}, m=0,1, \cdots, N$ respectively. In Eq. (A5), we have used the following relations according to Eq. (4)

$$
\begin{aligned}
& \frac{\partial \rho_{\mathrm{t}} \overline{M^{m}}}{\partial \rho_{i}}=M_{i}^{m} \\
& \delta \rho_{\mathrm{c}} \overline{M^{m}}=\sum_{i=1}^{K} M_{i}^{m} \delta \rho_{i} .
\end{aligned}
$$

Eqs. (A6a) and (A6b) follow because for a defined number of virtual discrete components to represent the continuous "component", $M_{i}$ is a constant. For example, we suppose that we regard the paraffins as a continuous "component" where $M$ is the number of carbon atoms in a molecule varying from 1 to 20 and $i$ represents the ordinal number corresponding to this defined separation of continuous "component". If we split the continuous distribution of parrafins into 190 virtual components, then, for example $i=15$ represents a virtual component which has 2.5 carbon atoms in a molecule, i.e. $M_{i}=2.5$ for $i=15$.

Multiply the $i+1$-th row of Eq. (A4) by $\rho_{i} M_{i}^{n}, n=0,1,2, \cdots, \infty$. We obtain with the help of Eq. (A6) the equation

$$
\begin{aligned}
& R T M_{i}^{n} \delta \rho_{i}+\sum_{m=0}^{N} f_{\mathrm{s} m}^{\mathrm{r}} \rho_{i} M_{i}^{m+n} \delta \rho_{\mathrm{s}}+\sum_{j=1}^{K} \sum_{m, l=0}^{N} f_{m l}^{\mathrm{r}} \rho_{i} M_{i}^{m+n} M_{j}^{l} \delta \rho_{j} \\
= & R T M_{i}^{n} \delta \rho_{i}+\sum_{m=0}^{N} f_{\mathrm{s} m}^{\mathrm{r}} \rho_{i} M_{i}^{m+n} \delta \rho_{\mathrm{s}}+\sum_{m, l=0}^{N} f_{m l}^{\mathrm{r}} \rho_{i} M_{i}^{m+n} \delta \rho_{\mathrm{t}} \overline{M^{l}}=0 .
\end{aligned}
$$


Summing up the second equality of Eq. (A7) with respect to $i$ from 1 to $K$ and multiplying by $\rho_{\mathrm{t}}^{-1}$, we obtain

$$
\frac{R T}{\rho_{\mathrm{t}}} \delta \rho_{\mathrm{t}} \overline{M^{n}}+\sum_{m=0}^{N} f_{\mathrm{s} m}^{\mathrm{r}} \overline{M^{m+n}} \delta \rho_{\mathrm{s}}+\sum_{m, l=0}^{N} f_{m l}^{\mathrm{r}} \overline{M^{m+n}} \delta \rho_{\mathrm{t}} \overline{M^{l}}=0 ; \quad n=0,1,2, \cdots
$$

Combining the second equality of Eq. (A5) with Eq. (A8) and arranging these equations in an order such that Eq. (A5) is the first, Eq. (A8) with $n=0$ is the second, Eq. (A8) with $n=1$ is the third and so on, we obtain an infinite-dimensional linear system of vector $\mathbf{v}$

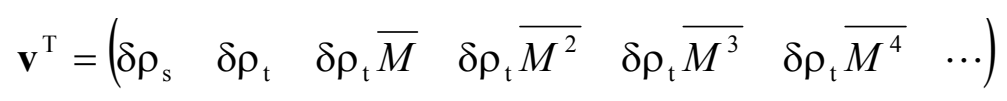

where superscript $T$ represents the matrix transpose. We denote the coefficient matrix of this infinite-dimensional linear system by A. According to Eq. (A5, A8), we find that the coefficients in $\mathbf{A}$ concerned with $\delta \rho_{\mathrm{t}} \overline{M^{l}}(l>N)$ are all $R T \rho_{\mathrm{t}}^{-1}$. The elements of $\mathbf{A}$ have the properties as following

$$
\left\{\begin{array}{c}
A_{i j}=0, \quad j>N+2 \text { and } j \neq i \\
A_{i i}=R T \rho_{\mathrm{t}}^{-1}, \quad i>N+2
\end{array} .\right.
$$

The structure of $\mathbf{A}$ is

$$
\mathbf{A}=\left(\begin{array}{ccccccc}
\times & \cdots & \times & & & \\
\vdots & \vdots & \vdots & & & & \\
\times & \cdots & \times & & & \\
\times & \cdots & \times & \times & & & \\
\times & \cdots & \times & & \times & & \\
\times & \cdots & \times & & & \times & \\
\vdots & \vdots & \vdots & & & & \ddots
\end{array}\right)
$$

where $\times$ represents the non-zero term; the blank parts are all zero. The first $N+2$ rows of matrix A are concerned only with the first $N+2$ components of vector $\mathbf{v}$ and form a square matrix; they are given by 


$$
\mathbf{A}_{(N+2) \times(N+2)}=\left(\begin{array}{ccccc}
j_{\mathrm{s}}+f_{\mathrm{ss}}^{\mathrm{r}} & f_{\mathrm{s} 0}^{\mathrm{r}} & f_{\mathrm{s} 1}^{\mathrm{r}} & \cdots & f_{\mathrm{s} N}^{\mathrm{r}} \\
\sum_{m=0}^{N} f_{\mathrm{s} m}^{\mathrm{r}} \overline{M^{m}} & j_{\mathrm{t}}+\sum_{m=0}^{N} f_{m 0}^{\mathrm{r}} \overline{M^{m}} & \sum_{m=0}^{N} f_{m 1}^{\mathrm{r}} \overline{M^{m}} & \cdots & \sum_{m=0}^{N} f_{m N}^{\mathrm{r}} \overline{M^{m}} \\
\sum_{m=0}^{N} f_{\mathrm{s} m}^{\mathrm{r}} \overline{M^{m+1}} & \sum_{m=0}^{N} f_{m 0}^{\mathrm{r}} \overline{M^{m+1}} & j_{\mathrm{t}}+\sum_{m=0}^{N} f_{m 1}^{\mathrm{r}} \overline{M^{m+1}} & \cdots & \sum_{m=0}^{N} f_{m N}^{\mathrm{r}} \overline{M^{m+1}} \\
\vdots & \vdots & \vdots & \ddots & \vdots \\
\sum_{m=0}^{N} f_{\mathrm{s} m}^{\mathrm{r}} \overline{M^{m+N}} & \sum_{m=0}^{N} f_{m 0}^{\mathrm{r}} \overline{M^{m+N}} & \sum_{m=0}^{N} f_{m 1}^{\mathrm{r}} \overline{M^{m+N}} & \cdots & j_{\mathrm{t}}+\sum_{m=0}^{N} f_{m N}^{\mathrm{r}} \overline{M^{m+N}}
\end{array}\right)
$$

where $j_{\mathrm{s}}=R T \rho_{\mathrm{s}}^{-1}, j_{\mathrm{t}}=R T \rho_{\mathrm{t}}^{-1}$ and the subscript $(N+2) \times(N+2)$ represents the first $N+2$ columns and rows of matrix $\mathbf{A}$. The sufficient and necessary condition for which $\mathbf{A v}=\mathbf{0}$ has a nontrivial solution is that the determinant of the coefficient matrix is zero, i.e.,

$$
\operatorname{det} \mathbf{A}=\left(R T \rho_{\mathrm{t}}^{-1}\right)^{L} \operatorname{det} \mathbf{A}_{(N+2) \times(N+2)}=0
$$

where $L$ is an arbitrary integer. Because we use all moment orders of the distribution in Eq. (A8) and because a set of moments uniquely determines a distribution [27], therefore the $2^{\text {nd }}$-order variation of the free energy includes all possible variations of the distribution function. As a result, Eq. (A13) is equivalent to Eq. (A4). The spinodal criterion is given by

$$
F_{\text {sp }}=\operatorname{det} \mathbf{A}_{(N+2) \times(N+2)}=0
$$

where subscript sp represents "spinodal".

\section{Derivation of Critical Criterion}

According to stability theory, the critical criterion is that the $3^{\text {rd }}$-order variation of the free energy per unit volume vanishes.

$$
\delta^{3} f=0
$$

This is equivalent to

$$
\delta F_{\mathrm{sp}}=0
$$

From Eq. (A12), $F_{\text {sp }}$ is a function of $\rho_{\mathrm{s}}$ and $\rho_{\mathrm{t}} \overline{M^{m}}, m=0,1, \cdots, 2 N$. Eq. (A16) can be written in the form

$$
\delta F_{\mathrm{sp}}=\frac{\partial F_{\mathrm{sp}}}{\partial \rho_{\mathrm{s}}} \delta \rho_{\mathrm{s}}+\sum_{m=0}^{2 N} \frac{\partial F_{\mathrm{sp}}}{\partial \rho_{\mathrm{t}} \overline{u^{m}}} \delta \rho_{\mathrm{t}} \overline{M^{m}}=0 .
$$


At the critical point, the spinodal criterion must also be satisfied. Thus the variation of $\rho_{\mathrm{s}}$ and $\rho_{\mathrm{t}} \overline{M^{m}}, m=0,1, \cdots, 2 N$ must satisfy the equation $\mathbf{A v}=\mathbf{0}$ where $\mathbf{A}$ and $\mathbf{v}$ are given by Eqs. (A11) and (A9) respectively. Similar to Heideman's work [18], we solve the singular linear system $\mathbf{A v}=\mathbf{0}$ to obtain at least one nontrivial value of vector $\mathbf{v}=\left(\begin{array}{llll}\delta \rho_{\mathrm{s}} & \delta \rho_{\mathrm{t}} & \cdots & \delta \rho_{\mathrm{t}} \overline{M^{2 N}}\end{array}\right)$ and substitute the solution into Eq. (A17). If Eq. (A17) is not satisfied, then this point is not a critical point.

A similar procedure uses another equivalent equation. Combining Eq. (A17) with the first $2 N+2$ rows of Eq. (A8), we obtain another linear system

$$
\left(\begin{array}{cccc}
A_{11} & A_{12} & \cdots & A_{1,2 N+2} \\
A_{21} & A_{22} & \cdots & A_{2,2 N+2} \\
\vdots & \vdots & \ddots & \vdots \\
A_{2 N+2,1} & A_{2 N+2,1} & \cdots & A_{2 N+2,2 N+2} \\
\frac{\partial F_{\mathrm{sp}}}{\partial \rho_{\mathrm{s}}} & \frac{\partial F_{\mathrm{sp}}}{\partial \rho_{\mathrm{t}}} & \cdots & \frac{\partial F_{\mathrm{sp}}}{\partial \rho_{\mathrm{t}} \overline{M^{2 N}}}
\end{array}\right)\left(\begin{array}{c}
\delta \rho_{\mathrm{s}} \\
\delta \rho_{\mathrm{t}} \\
\vdots \\
\delta \rho_{\mathrm{t}} \overline{M^{2 N}}
\end{array}\right)=0
$$

where $A_{i j}, i, j \leq 2 N+2$ are given by Eq. (16). A critical point must satisfy the condition that Eq. (A18) has a nontrivial solution for the vector of variations. Since only the first $2 N+2$ rows of A are concerned with $\rho_{\mathrm{s}}$ and $\rho_{\mathrm{t}} \overline{M^{m}}, m=0,1, \cdots, 2 N$, other rows with variations of higher order moments $(m>2 N)$ are of no concern. The first $N+2$ rows in Eq. (A18) are linearly dependent because they satisfy Eq. (A14). At a first-order critical point, $N+1$ rows among them are linearly independent. However, we do not know which of these are linearly independent. Because component $\mathrm{s}$ is discrete, the $1^{\text {st }}$ row of Eq. (A18) is independent of the others. The linear dependent row is somewhere between the $2^{\text {nd }}$ row and the $N+2$-th row. For simplicity, we multiply the $2^{\text {nd }}$ line by $-\bar{M}$ and add it to the $3^{\text {rd }}$ line and then cancel the $2^{\text {nd }}$ line. The critical criterion is given by

$$
F_{\text {cr }}=\left|\begin{array}{cccc}
A_{11} & A_{12} & \cdots & A_{1,2 N+2} \\
A_{31}-\bar{M} A_{21} & A_{32}-\bar{M} A_{22} & \cdots & A_{3,2 N+2}-\bar{M} A_{3,2 N+2} \\
\vdots & \vdots & \ddots & \vdots \\
A_{2 N+2,1} & A_{2 N+2,2} & \cdots & A_{2 N+2,2 N+2} \\
\frac{\partial F_{\mathrm{sp}}}{\partial \rho_{\mathrm{s}}} & \frac{\partial F_{\mathrm{sp}}}{\partial \rho_{\mathrm{c}}} & \cdots & \frac{\partial F_{\mathrm{sp}}}{\partial \rho_{\mathrm{c}} \overline{M^{2 N}}}
\end{array}\right|=0
$$


where subscript cr represent "critical".

From inspection of Eq. (A12), we can directly generalize Eq. (A12) to the spinodal criterion for a system containing $K$ discrete components and one continuous component. It is given by

$$
F_{\text {sp }}=\operatorname{det} \mathbf{A}
$$

where matrix $\mathbf{A}$ is given by

$$
\mathbf{A}=\left(\begin{array}{cccccc}
j_{\mathrm{S}_{1}}+f_{\mathrm{S}_{1} \mathrm{~S}_{1}}^{\mathrm{r}} & \cdots & f_{\mathrm{S}_{1} \mathrm{~S}_{K}}^{\mathrm{r}} & f_{\mathrm{S}_{1} 0}^{\mathrm{r}} & \cdots & f_{\mathrm{S}_{1} N}^{\mathrm{r}} \\
\vdots & \ddots & \vdots & \vdots & \vdots & \vdots \\
f_{\mathrm{S}_{K} \mathrm{~S}_{1}}^{\mathrm{r}} & \cdots & j_{\mathrm{S}_{K}}+f_{\mathrm{S}_{K} \mathrm{~S}_{K}}^{\mathrm{r}} & f_{\mathrm{S}_{K} 0}^{\mathrm{r}} & \cdots & f_{\mathrm{S}_{K} N}^{\mathrm{r}} \\
\sum_{m=0}^{N} f_{\mathrm{S}_{1} m}^{\mathrm{r}} \overline{M^{m}} & \cdots & \sum_{m=0}^{N} f_{\mathrm{S}_{K^{m}}}^{\mathrm{r}} \overline{M^{m}} & j_{\mathrm{c}}+\sum_{m=0}^{N} f_{0 m}^{\mathrm{r}} \overline{M^{m}} & \cdots & \sum_{m=0}^{\mathrm{r}} f_{N m}^{\mathrm{r}} \overline{M^{m}} \\
\vdots & \vdots & \vdots & \vdots & \ddots & \vdots \\
\sum_{m=0}^{N} f_{\mathrm{S}_{1} m}^{\mathrm{r}} \overline{M^{m+N}} & \cdots & \sum_{m=0}^{N} f_{\mathrm{S}_{K} m}^{\mathrm{r}} \overline{M^{m+N}} & \sum_{m=0}^{N} f_{0 m}^{\mathrm{r}} \overline{M^{m+N}} & \cdots & j_{\mathrm{c}}+\sum_{m=0}^{N} f_{N m}^{\mathrm{r}} \overline{M^{m+N}}
\end{array}\right)
$$

where $K$ is the number of discrete components; subscript $\mathrm{s}_{i}$ means the discrete component $i$; $f_{\mathrm{S}_{i}}^{\mathrm{r}}$ is the derivative of the residual free energy with respect to molar density of discrete component $i ; f_{m}^{\mathrm{r}}$ is the derivative of the residual free energy per unit volume with respect to moment $\overline{M^{m}}$. The derivation of the critical criterion is similar to the generalization given by Eq. (A12) to Eq. (A21). For the stability criteria of a system that contains several continuous "components", the derivation is the same but tedious.

\section{Literature Cited}

1. R. Aris and G. R. Gavalas, R. Soc. London. Ser. A, 260 (1966) 351-411.

2. J. J. Salacuse and G. Stell, J. Chem. Phys., 77 (1982) 3714-3725.

3. J. A. Gualtieri, J. M. Kincaid, G. Morrison, J. Chem. Phys. 77 (1982) 521-536.

4. J. G. Briano and E. D. Glandt, Fluid Phase Equilibria, 14 (1983) 91-102.

5. M. T. Ratzsch and H. Kehlen, Fluid Phase Equilibria, 14(1983)225-234.

6. H. Kehlen, M. Ratzsch, J. Bergmann, AIChE J., 31(1985)1136-1148.

7. R. L. Cotterman and J. M. Prausnitz, in G. Astarita and S. I. Sandler (Eds.) Kinetic and Thermodynamic Lumping of Multicomponent Mixtures, Elsevier, Amsterdam, 1991, pp 229275. 
8. K. A. Johnson, D. A. Jonah, J. M. Kincaid and G. Morrison, J. Chem. Phys. 82 (1985) 51785183.

9. J. M. Kincaid, R. A. McDonald and G. Morrison, J. Chem. Phys. 87 (1987) 5425-5736.

10. R. L. Cotterman, R. Bender and J. M. Prausnitz, Ind. Eng. Chem. Process Des. Dev., 24 (1985) 194-203.

11. D. Browarzik and H. Kehlen, Fluid Phase Equilibria, 123 (1996) 17-28.

12. D. Browarzik, M. Kowalewski and H. Kehlen, Fluid Phase Equilibria, 142 (1998) 149-162.

13. Y. Hu and X. Ying, Fluid Phase Equilibria, 127 (1997) 21-27.

14. Y. Hu and J. M. Prausnitz, Fluid Phase Equilibria, 130 (1997) 1-18.

15. J. Cai, H. Liu, Y. Hu and J. M. Prausnitz, Fluid Phase Equilibria, 168 (2000) 91-106.

16. L. Bellier-Castella, H. Xu and M. Baus, J. Chem. Phys., 113 (2000) 8337-8347.

17. J. Jiang and J. M. Prausnitz, J. Chem. Phys., 111 (1999) 5964-5974.

18. R. A. Heidemann and A. M. Khalil, AIChE J., 26 (1980) 769-779.

19. B. E. Poling, J. M. Prausnitz and J. P. O'Connell, The Properties of Gases and Liquids, 5th edition, McGraw-Hill Book Company, New York, 2000.

20. C. P. Hicks and C. L. Young, J. Chem. Soc. Faraday Trans. II, 73(1977)597-622

21. P. H. van Konynenburg and R. L. Scott, Philos. Trans. Roy. Soc. London A, 298(1980)495540

22. J. S. Rowlinson, Liquids and Liquids Mixtures, Butterworth Scientific, London, 1982

23. C. P. Hicks and C. L. Young, Chem. Rev., 75(1975)119-175.

24. Y. Lin, R. Chen, P. Chappelear and R. Kobayashi, J. Chem. Eng. Data, 22(1977)402-408.

25. R. Chen, P. Chappelear and R. Kobayashi, J. Chem. Eng. Data, 21(1976)213-219.

26. See, e.g. J. Tester and M. Modell, Thermodynamics and its Applications, $3^{\text {rd }}$ edition, Prentice Hall, 1997.

27. See any college text books about probability. 